\title{
Open Rotor Computational Aeroacoustic Analysis with an Immersed Boundary Method
}

\author{
Christoph Brehm*1 \\ ${ }^{1}$ Science and Technology Corporation, Moffett Field, CA 94035 \\ Michael F. Barad ${ }^{* 2}$, and Cetin C. Kiris ${ }^{\dagger 2}$ \\ ${ }^{2}$ NASA Ames Research Center, Moffett Field, CA 94035
}

\begin{abstract}
Reliable noise prediction capabilities are essential to enable novel fuel efficient open rotor designs that can meet the community and cabin noise standards. Toward this end, immersed boundary methods have reached a level of maturity so that they are being frequently employed for specific real world applications within NASA. This paper demonstrates that our higher-order immersed boundary method provides the ability for aeroacoustic analysis of wake-dominated flow fields generated by highly complex geometries. This is the first of a kind aeroacoustic simulation of an open rotor propulsion system employing an immersed boundary method. In addition to discussing the peculiarities of applying the immersed boundary method to this moving boundary problem, we will provide a detailed aeroacoustic analysis of the noise generation mechanisms encountered in the open rotor flow. The simulation data is compared to available experimental data and other computational results employing more conventional CFD methods. The noise generation mechanisms are analyzed employing spectral analysis, proper orthogonal decomposition and the causality method.
\end{abstract}

\section{Introduction}

There has been a renewed interest in pursuing contra-rotating open rotor (CROR) propulsion technology due to the large potential of significantly reducing fuel consumption. A main concern for the design of such systems is that they must meet community noise and cabin noise standards. Hence, providing reliable noise prediction capabilities for contra-rotating open rotors is essential in the design of low-noise rotor propulsion systems. In recent years, NASA initiated several research efforts that have assessed the capabilities on noise source prediction and analysis of contra-rotating open rotors. Several low to high fidelity simulation tools were used to provide noise predictions and their results were compared to experimental data. ${ }^{1-5}$ One of the key challenges is to conduct efficient high-fidelity simulations of this relatively complex geometry including moving boundaries.

While providing a detailed acoustic analysis of the noise generation mechanisms, a key contribution of this paper is to conduct this analysis by employing a higher-order accurate immersed boundary method. This is the first ever application of an immersed boundary method for noise prediction of an open rotor system. It will be demonstrated that this method provides a reliable noise prediction capability. Immersed Boundary Methods (IBMs) have been developed for many years and have appeared in various forms since they were first introduced by Peskin ${ }^{6,7}$ (see for example Goldstein et al. ${ }^{8}$ LeVeque and Li, ${ }^{9}$ Wiegmann and Bube, ${ }^{10}$ Linnick and Fasel, ${ }^{11}$ Johansen and Collela, ${ }^{12}$ Mittal and Iaccarino, ${ }^{13}$ Zhong, ${ }^{14}$ Duan et al. ${ }^{15}$ and many others). These methods were first introduced as a nontraditional approach for numerically solving initial/boundary-value problems for complex geometries on Cartesian meshes and have matured to become increasingly important for a wide range of applications. ${ }^{16-18}$ One of the key advantages of immersed boundary methods is that the computational meshes can be automatically be generated starting from a water tight surface triangulation independent of the complexity of the geometry. The grid-generation process for body-fitted structured or

*Research Scientist, Applied Modeling and Simulation Branch, NAS Division, MS N258-2 and AIAA Senior Member

${ }^{\dagger}$ Branch Chief, Applied Modeling and Simulation Branch, NAS Division, MS N258-2 and AIAA Senior Member 
unstructured grids for complex geometries is generally very laborious because the process aims at generating well behaved grids that have sufficient local resolution while minimizing the total number of required grid points. Except for simple geometries, these conflicting requirements can lead to deterioration in grid quality, thereby negatively impacting the accuracy and convergence properties of the flow solver. For highly complex geometries, which are relevant for many fields of science and engineering, the process of generating a highquality grid is extremely time consuming. For flows involving moving and deforming boundaries, IBMs also provide clear advantages over classical body-fitted grid approaches. Simulating such flows on body-fitted grids requires generating a new grid at each time step and a procedure to project the solution onto this new grid (Tezduyar ${ }^{19}$ ). These two characteristics (grid deformation and projection of solution) for body-fitted grids associated with simulating flows with moving and deforming boundaries may negatively impact the accuracy, robustness, and computational cost of the numerical solution method. Particularly, in cases where the boundary exhibits large motions, body-conformal grid strategies cause immense difficulties in the solution procedure. This is where an immersed boundary approach with its fully Eulerian approach provides great advantages. IBMs provide a much more convenient way of including the body motion and deformation by using a stationary non-deforming Cartesian grid. While these methods simplify the grid generation process, a detailed mathematical understanding of the IBM is necessary to avoid a negative impact of the boundary treatment on the robustness, the convergence behavior and the accuracy of the numerical scheme.

Immersed boundary methods also posses well known shortcomings that have limited their applicability to wider range of applications. Most IBMs are only lower-order accurate and suffer from robustness issues when extended to higher-order. The near wall accuracy is, however, critical when simulating laminar to turbulent transition scenarios for wall-bounded flows to capture relevant instability mechanisms. Higherorder immersed boundary methods have been presented in a number of research studies by Linnick and Fasel, ${ }^{11}$ Zhong, ${ }^{14}$ Duan et al. ${ }^{15}$ Brehm and Fasel, ${ }^{20}$ Brehm et al.,${ }^{21}$ and others. The current method employed within NASA's LAVA CFD solver ${ }^{16}$ is based on the higher-order immersed boundary method discussed in Brehm et al. ${ }^{21}$

The current abstract provides a rough idea of what can be expected in the final paper. The abstract proceeds as follows: section II introduces the numerical scheme and immersed boundary method used to solve the compressible Navier-Stokes equations. Section III presents the geometry used in the simulation and provides an overview of the simulation setup. Section IV provides an initial unsteady flow visualization displaying some of the complicated flow features. Section V provides a preliminary comparison of the present CFD data obtained on a coarse grid with available experimental data acquired in NASA's 8-foot $\times$ 6 -foot wind tunnel. A preliminary acoustic analysis is provided in section VI employing proper orthogonal decomposition and spectral analysis of the pressure field in the acoustic near field. A brief description of the causality method is also provided in section VI that will be used to link the unsteady flow features in the noise source regions to the acoustic signal in the far-field. Finally, some preliminary conclusions and an outlook to the final paper is provided in section VII.

\section{Numerical Methods}

\section{II.A. Interior and Temporal Discretizations}

The compressible Navier-Stokes equations considering an ideal, Newtonian, non-reactive gas are

$$
\begin{gathered}
\frac{\partial \rho}{\partial t}+\nabla \cdot(\rho \boldsymbol{u})=0, \\
\frac{\partial \rho \boldsymbol{u}}{\partial t}+\nabla \cdot(\rho \boldsymbol{u u}+p \underline{\boldsymbol{\delta}}-\underline{\boldsymbol{\tau}})=0, \\
\frac{\partial E}{\partial t}+\nabla \cdot(E \boldsymbol{u}+\boldsymbol{u} \cdot(p \underline{\boldsymbol{\delta}}-\underline{\boldsymbol{\tau}})-\kappa \nabla T)=0, \quad \text { with } \\
E=\frac{p}{\gamma-1}+\frac{1}{2} \rho \boldsymbol{u} \cdot \boldsymbol{u} \quad \text { and } \quad p=\rho R T .
\end{gathered}
$$

In the above equations $\rho$ is the density, $\boldsymbol{u}$ is the velocity vector, $\underline{\boldsymbol{\delta}}$ is the unit tensor, $p$ is the static pressure, $T$ is the temperature, $E$ is the total energy, $\gamma$ is the specific heat ratio, $R$ is the gas constant, and $\kappa$ is the thermal conductivity. The viscous stress tensor is

$$
\underline{\boldsymbol{\tau}}=\mu(2 \underline{\boldsymbol{S}})+\left(\beta-\frac{2}{3} \mu\right)(\nabla \cdot \boldsymbol{u}) \underline{\boldsymbol{\delta}} .
$$


The dynamic viscosity is given by $\mu, \beta$ is the bulk viscosity, and the strain rate tensor $\underline{\boldsymbol{S}}$ is defined as $\underline{\boldsymbol{S}}=\frac{1}{2}\left(\nabla \boldsymbol{u}+(\nabla \boldsymbol{u})^{T}\right)$.

Equations (1)-(2) can be re-written in vector form as follows

$$
\frac{\partial \boldsymbol{W}}{\partial t}+\nabla \cdot\left(\overrightarrow{\mathcal{F}}-\overrightarrow{\mathcal{F}}_{v}\right)=0, \quad \text { with } \overrightarrow{\mathcal{F}}=\left(\boldsymbol{F}^{1}, \boldsymbol{F}^{2}, \boldsymbol{F}^{3}\right) \quad \text { and } \quad \overrightarrow{\mathcal{F}}_{v}=\left(\boldsymbol{F}_{v}^{1}, \boldsymbol{F}_{v}^{2}, \boldsymbol{F}_{v}^{3}\right) .
$$

The conservative variable vector $\boldsymbol{W}=(\rho, \rho u, \rho v, \rho w, \rho H-p)^{T}$ and the inviscid fluxes are

$$
\boldsymbol{F}^{1}=\left[\begin{array}{c}
\rho u \\
\rho u^{2}+p \\
\rho u v \\
\rho u w \\
\rho u H
\end{array}\right], \boldsymbol{F}^{2}=\left[\begin{array}{c}
\rho v \\
\rho v u \\
\rho v^{2}+p \\
\rho v w \\
\rho v H
\end{array}\right] \text {, and } \boldsymbol{F}^{3}=\left[\begin{array}{c}
\rho w \\
\rho w u \\
\rho w v \\
\rho w^{2}+p \\
\rho w H
\end{array}\right] \text {, }
$$

where the total enthalpy is $H=h+\vec{u} \cdot \vec{u} / 2$. The viscous fluxes are

$$
\boldsymbol{F}_{v}^{1}=\left[\begin{array}{c}
0 \\
\tau_{x x} \\
\tau_{x y} \\
\tau_{x z} \\
f_{v}^{1}
\end{array}\right], \boldsymbol{F}_{v}^{2}=\left[\begin{array}{c}
0 \\
\tau_{y x} \\
\tau_{y y} \\
\tau_{y z} \\
f_{v}^{2}
\end{array}\right], \text { and } \boldsymbol{F}_{v}^{3}=\left[\begin{array}{c}
0 \\
\tau_{z x} \\
\tau_{z y} \\
\tau_{z z} \\
f_{v}^{3}
\end{array}\right]
$$

The interior discretization of the convective terms follows the approach presented in Laible and Fasel. ${ }^{22}$ The forward-flux, $\mathbf{F}^{+}$, and the backward-flux, $\mathbf{F}^{-}$, are discretized with $n^{\text {th }}$-order accurate centered upwind finite differences as proposed by Zhong. ${ }^{23}$ As in Laible and Fasel, ${ }^{22}$ a parameter $\alpha$ is used to determine the degree of upwinding for the grid-centered upwind differences

$$
\left.\frac{\partial \phi}{\partial x}\right|_{i}=\sum_{k=i-N_{s}}^{i+N_{s}} c_{k} \phi_{k}-\left.\alpha \overline{\Delta x} \frac{\partial^{2 N_{s}-1} \phi}{\partial x^{2 N_{s}-1}}\right|_{i}
$$

where $c_{k}$ is the finite difference stencil coefficient, $\overline{\Delta x}$ is the averaged grid spacing over the spatial extent of the stencil and $N_{s}$ determines the number of grid points in the stencil. Note that a central difference stencil can be achieved by choosing $\alpha=0$. In this case a higher-order filter based on work by Visbal and Gaitonde ${ }^{24}$ is used to regularize the solution. In accordance to the chosen discretization in Laible and Fasel, ${ }^{22}$ the $n^{t h}$-order accurate upwind stencils are calculated with $n=9,7,5,3,1$ and $\alpha=-1500,72,-12,0.6,-1$. In the current paper, it is assumed that the numerical fluxes are approximated with flux vector splitting schemes, i.e., Lax Friedrichs, van Leer, AUSMPW+, etc. The compressible Navier-Stokes equations are advanced in time with an explicit fourth-order accurate Runge-Kutta scheme based on the Shu-Osher formulation. ${ }^{25}$ For accurately capturing flow discontinuities higher-order shock capturing schemes ${ }^{26}$ are also available. The computational domain is decomposed into block-structured sub-domains supporting adaptive-mesh refinement as well as generalized curvilinear coordinates. In order to maintain higher-order accuracy across coarse-fine block interfaces, up-to fourth-order accurate prolongation and restriction operators are available.

\section{II.B. Immersed Boundary Method}

In this section, the theoretical basis of the IIM presented in Brehm and Fasel ${ }^{20}$ is extended to the compressible Navier-Stokes equations. Many immersed methods have been developed in the past; however, in the derivation of these schemes, usually only the order of the local truncation error or accuracy of the numerical scheme has been considered. A posteriori, the numerical stability of these schemes is commonly demonstrated (in a global sense) by considering a number of different test-problems, and in a few cases, an additional global matrix stability analysis is employed (see for example Zhong ${ }^{14}$ ). The basis of the IIM by Brehm and Fasel $^{20}$ is that the stencil coefficients are locally optimized in order to improve the stability of the scheme.

The notion of developing a strategy for improving the spectral properties of immersed methods originated from the idea that the stability of the numerical scheme can be formulated as an $N$-dimensional optimization 
problem, where $N$ represents the number of irregular grid points. Instead of solely considering the local truncation error in the derivation of the finite-difference grid stencil at an irregular grid point, an overdetermined system of equations is constructed to determine the stencil coefficients. We employed an overdetermined system of equations because it allows one to select free parameters, which can be tuned towards optimizing the local stability properties of the finite-difference stencil. Thus, assuming one free parameter per irregular grid point an $N$-dimensional optimization problem for $N$ irregular grid points is obtained.

The objective function of the optimization problem must be carefully chosen and depends on the nature of the particular partial-differential equation of interest. For advection-diffusion-type equations, the spectral radius of the update matrix may be considered an appropriate objective function. In the current paper, we refer to the update matrix as the matrix which updates the solution from time $t_{n}$ to the next time-step, $t_{n+1}$. The update matrix, therefore, contains information about the spatial and temporal discretization of the numerical scheme. The spectral radius is well-suited in this situation, because its actual value is associated with the stability of the numerical scheme and it can be used to formulate a necessary condition for the stability of the update matrix. For non-linear systems of equations, a linearization is necessary prior to applying the outlined linear stability analysis concepts. Note that the immersed boundary method used in this paper solely uses linear stability theory and, therefore, neglects possible non-linear effects on the stability.

In principal, it is possible to solve a coupled $N$-dimensional optimization problem for $N$ irregular grid points. From a practical point of view, however, it is not very efficient or desirable to solve such a large system of equations, especially for FSI problems where the grid topology is time dependent, thereby requiring this procedure to be repeated at each time-step. Therefore, in the current approach, we aimed at isolating the boundary stencils from the remainder of the computational domain so that the optimization problem can be formulated for each irregular grid-point separately. Instead of solving a global $N$-dimensional optimization problem we solve 1D optimization problems at each of the $N$ irregular grid point individually. This approach greatly reduces the computational effort needed to determine the "optimal" stencil coefficients. We refer to the aspect of turning the global $N$-dimensional problem into $N$ one-dimensional problems as "localization" or "localization assumption". In Brehm and Fasel, ${ }^{20}$ it was demonstrated, both numerically and analytically, that for advection-diffusion type equations, the localization assumption appears to be valid as long as the von Neumann number does not reach a limiting value. For a more detailed discussion on the original approach, the interested reader is referred to Brehm and Fasel. ${ }^{20}$ In the following discussion, the basic steps for extending the IIM for the compressible Navier-Stokes equations are outlined.

The main difference for the application of the IIM to the compressible Navier-Stokes equations instead of the incompressible Navier-Stokes equations (solved by utilizing the approximate projection method) is the use of numerical fluxes. Flux vector splitting schemes, i.e. van Leer ${ }^{27}$ Rusanov, and AUSMPW+, are adopted here. In the flux vector splitting approach, the convective flux is divided into a forward-flux and a backward-flux, $\mathbf{F}=\mathbf{F}^{-}+\mathbf{F}^{+}$. The flux vector splitting approach is also applied at irregular grid points, which can be employed to stabilize the irregular grid stencils. Assuming subsonic flow speeds in the vicinity of the immersed boundary (no slip wall), upwinding can always be applied in the upwind direction towards the wall. This is in contrast to the scalar advection equation where the advection speed determines the upwind direction. The basic ideas of the IIM implementation are discussed for the 1D Euler equations in conservative variable formulation,

$$
\left(\begin{array}{c}
\rho \\
\rho u \\
E
\end{array}\right)_{t}=\left(\begin{array}{c}
\rho u \\
\rho u^{2}+p \\
u(E+p)
\end{array}\right)_{x},
$$

where the total energy can be expressed as $E=\rho\left(T /\left((\gamma-1) \gamma M^{2}\right)+u^{2} / 2\right)$. The fluid is assumed to be an ideal gas, which can be modeled by the equation of state. Applying the flux vector splitting approach to the 1D Euler equations leads to

$$
\mathbf{W}_{t}=\mathbf{F}_{x}=\mathbf{F}_{x}^{-}+\mathbf{F}_{x}^{+},
$$

where the solution vector can be written as $\mathbf{W}=\left(w_{1}, w_{2}, w_{3}\right)^{T}=(\rho, \rho u, E)^{T}$. In order to numerically analyze the 1D Euler system in Equation (6), the Jacobian, $\partial \mathbf{F} / \partial \mathbf{W}$, is introduced,

$$
\mathbf{W}_{t}=\frac{\partial \mathbf{F}}{\partial \mathbf{W}} \cdot \frac{\partial \mathbf{W}}{\partial x}=\frac{\partial \mathbf{F}^{-}}{\partial \mathbf{W}} \cdot \frac{\partial \mathbf{W}}{\partial x}+\frac{\partial \mathbf{F}^{+}}{\partial \mathbf{W}} \cdot \frac{\partial \mathbf{W}}{\partial x} .
$$


This leads to an equation including the derivatives of the forward/backward-flux vectors, $\mathbf{F}^{ \pm}$, with respect to the solution vector $\mathbf{W}$. The gradient of the convective flux vectors, $\mathbf{F}^{ \pm}$, is discretized with the discrete forward difference operator, $\underline{\mathbf{D}}_{x}^{+}$, (for the forward-flux) and in backward direction with the discrete backward difference operator, $\underline{\mathbf{D}}_{x}^{-}$, (for the backward-flux),

$$
\begin{aligned}
\mathbf{W}_{t} & =\frac{\partial \mathbf{F}^{+}}{\partial \mathbf{W}} \cdot \frac{\partial \mathbf{W}}{\partial x}+\frac{\partial \mathbf{F}^{-}}{\partial \mathbf{W}} \cdot \frac{\partial \mathbf{W}}{\partial x} \\
& \cong \frac{\partial \mathbf{F}^{+}}{\partial \mathbf{W}} \cdot\left(\underline{\mathbf{D}}_{x}^{+} \cdot \mathbf{W}\right)+\frac{\partial \mathbf{F}^{-}}{\partial \mathbf{W}} \cdot\left(\underline{\mathbf{D}}_{x}^{-} \cdot \mathbf{W}\right) .
\end{aligned}
$$

difference operators, $\underline{\mathbf{D}}_{x}^{ \pm}$, are provided in Brehm et al. ${ }^{21}$ For an irregular grid point with an immersed boundary located to the left, the optimization procedure is only applied to the differentiation of the forward flux and to the differentiation of the backward flux when the immersed boundary located to the right. The $n^{\text {th }}$-order accurate finite difference operator for the differentiation of the backward flux is obtained by utilizing $n+2$ grid points (including the boundary point) and applying a least-squares procedure to determine the stencil coefficients for the over-determined system of equations. In order to obtain pressure and temperature boundary conditions a weighted-least-squares interpolation approach is utilized. More details about the basics of the immersed boundary method can be found in Brehm et al. ${ }^{20,21}$

\section{Simulation Setup}

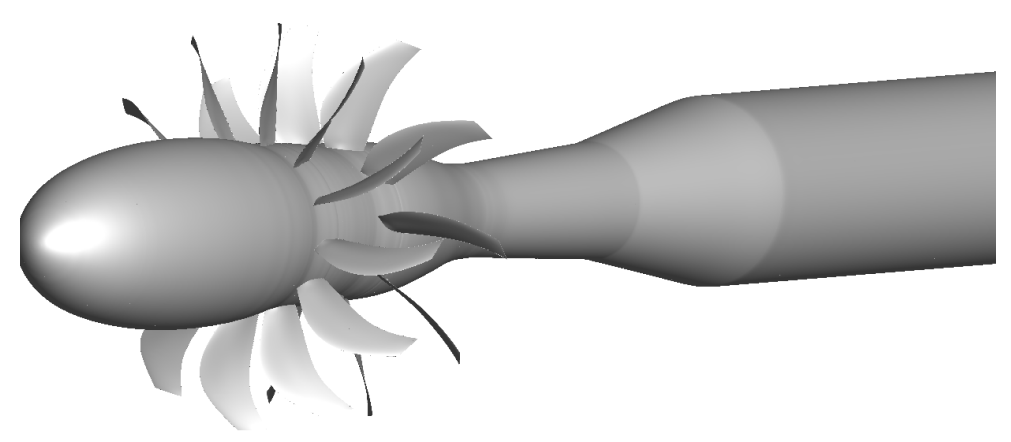

Figure 1. Un-installed open rotor configuration used in the present CFD analysis.

Figure 1 presents the geometry used in the present CFD simulation. The CROR blade set shown in figure 1 is a relatively modern GE design, namely F31/A31, with 12 blades on the front rotor and 10 blades on the second rotor. The simulation data is compared to the high-speed aerodynamics and acoustic data that was obtained for a model scale geometry in NASA's 8 -foot $\times 6$-foot wind tunnel at cruise conditions. The front and aft rotor diameters are $0.66 \mathrm{~m}$ and $0.63 \mathrm{~m}$, respectively. The two rotors are separated by $0.2 \mathrm{~m}$. The aerodynamic and acoustic data were acquired for the rotors in un-installed configuration without fuselage and pylon at zero angle of attack.

A cut through the computational mesh employed for the current simulations is shown in Figures 2. Three grid resolutions are employed with 150, 250, and 500 million grid points to test the sensitivity of the simulation results with respect to the grid resolution. Note that all results shown in this abstract are based on the coarse grid simulations. Each Cartesian block (marked with solid black lines) contains $16^{3}$ grid points. Grid refinement is applied in regions where high unsteadiness and large gradients are expected. Since storing the entire volume data for each time-step requires excessively large memory, several sampling planes were used to gather the unsteady flow data at each time-step. Most of the unsteady flow analysis was applied to the data recorded in these sampling planes. Note that throughout this paper all flow quantities were normalized with the free stream flow quantities, i.e., free-stream velocity, $V_{\infty}$, pressure, $p_{\infty}$, and temperature, $T_{\infty}$. The Reynolds number based on blade diameter $D, V_{\infty}$, and $T_{\infty}$ is approximately $1.2 \times 10^{7}$. The free-stream Mach number is 0.78 . 


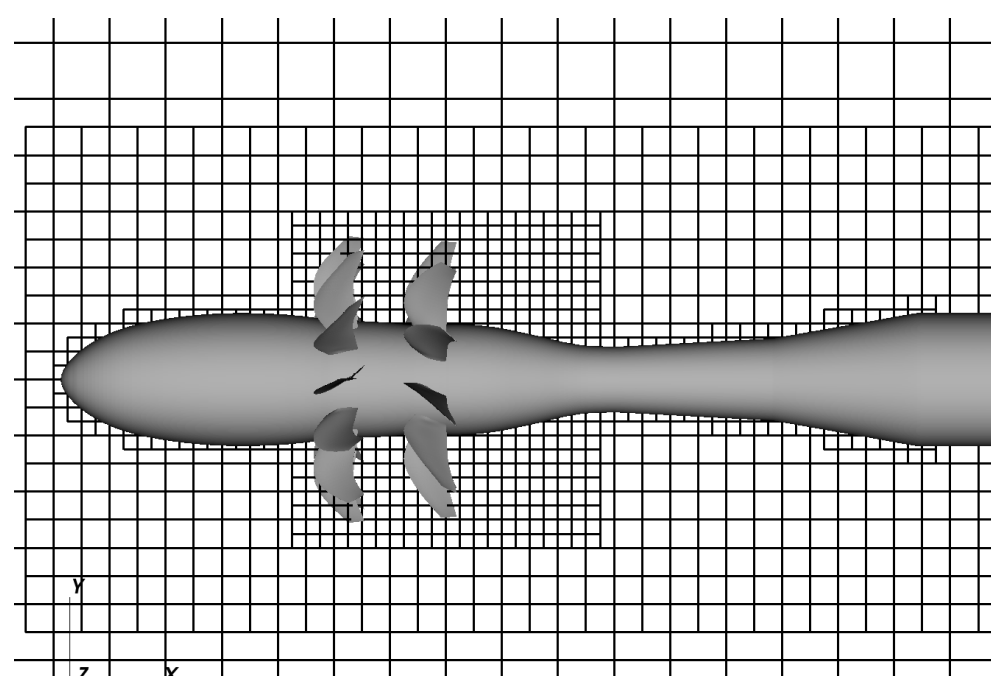

Figure 2. Computational mesh layout in the vicinity of the open rotor.

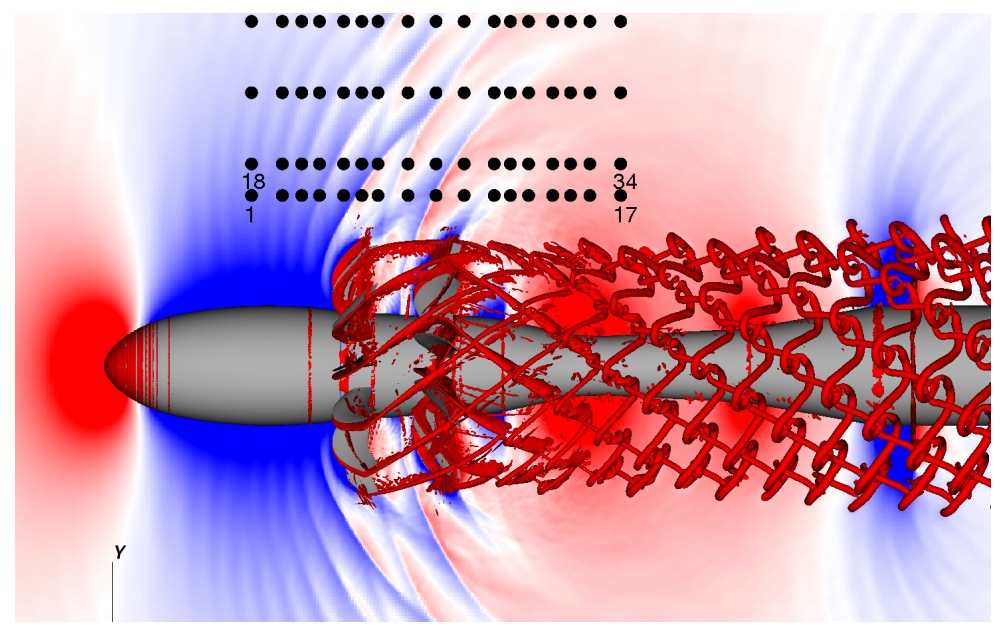

Figure 3. Colored gauge-pressure in the center plane and iso-surface of $Q$-criterion. The black dots mark the Kulite sensor locations where experimental data is available.

\section{Main Flow Features}

Color contours of gauge pressure and an iso-surface of $Q$-criterion is shown in figure 3 . The black dots mark the Kulite sensor locations where experimental data is available. The Kulite sensors are numbered from left-to-right and top-to-bottom in increasing order. On the front and aft rotors, tip-vortices are generated at the end of each blade. The tip-vortices generated at the front blade impinge on the aft rotor blade as well as interact with the tip-vortices of the second rotor. The color contours of gauge pressure nicely visualize strong pressure waves that are generated by each rotor blade. These distinct pressure waves will generate strong peaks in the frequency spectra at distinct blade-passing-frequencies (BPF). One of the key challenges for simulating this flow field is to also capture broadband noise that is generated by the finer-scale turbulence in the wake. For lower rotor speeds, the broadband noise component becomes more important in comparison to the tonal noise and, thus, an accurate prediction is required. In order to capture the broadband noise, sufficient grid resolution is required to resolve the wide range of temporal and spatial scales in the turbulent wake. 


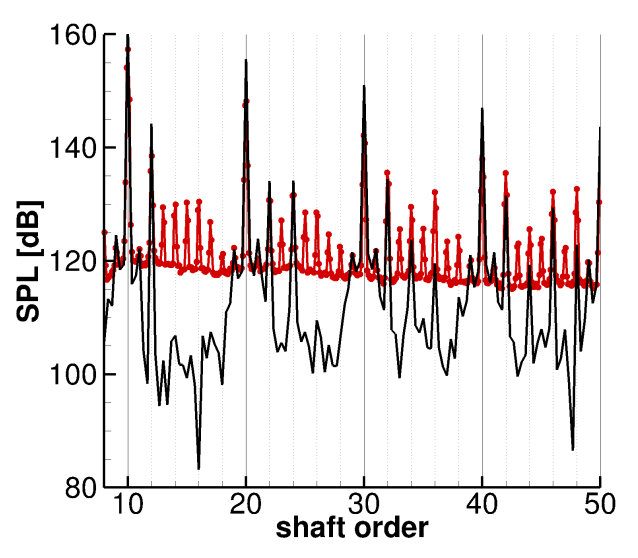

(a)

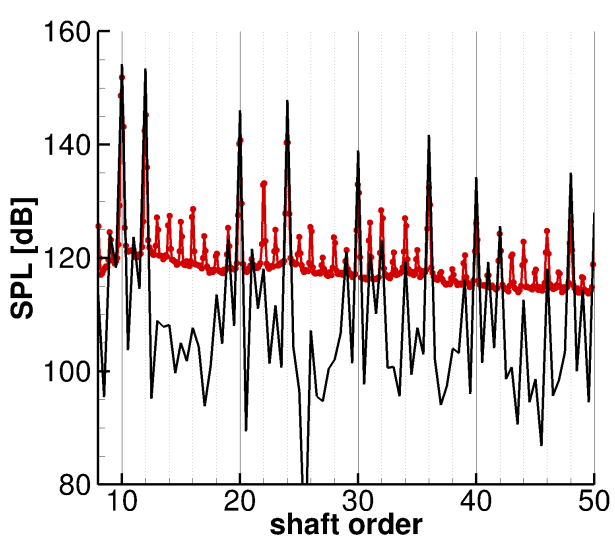

(b)

Figure 4. Sound pressure level (SPL) spectra at Kulites (a) \#9 and (b) \#10 located at a vertical distance of $0.43 \mathrm{~m}$. Experimental data is shown with red lines and coarse grid computations with black lines.

\section{Preliminary Comparison with Experimental Data}

Figures $4 \mathrm{a}$ and $4 \mathrm{~b}$ show a preliminary comparison of the coarse grid CFD results with experimental data obtained at different Kulite locations. It is expected that the coarse-grid resolution is not sufficient to capture some of the higher-order interactions. Due to insufficient grid resolution the large tip vortices cannot break up as quickly and, therefore, some of the distinct tones are slightly over-predicted in the spectra. This result is consistent with the observations by Housman et al. utilizing a conventional overset approach to simulate the flow around the open rotor.

\section{Acoustic Analysis}

The following discussion will provide a brief overview of the acoustic analysis tools that will be used in the final version of the paper to identify the dominant noise sources and study the noise generation mechanisms. Some preliminary results are provided.

\section{VI.A. Proper Orthogonal Decomposition}

POD decomposes the flow field into a set of orthogonal basis functions that capture most of the flow energy in terms of a user-defined norm with the least number of modes. ${ }^{28}$ In many fluid dynamics applications, POD is used to identify energetic flow structures. A dynamical significance of existing POD modes is not guaranteed. Freund and Colonius ${ }^{29}$ applied POD to a Mach 0.9 turbulent jet. and noted that the sound radiated by turbulence amounts to a very small fraction of the total energy. Thus, a POD approach in the temporal domain may not be able to capture the physics of the radiated sound waves. When analyzing the acoustic field it may be advantageous to follow an approach similar to the one described in Suzuki et $a l .{ }^{30}$ Instead of using snapshots in time, Suzuki et al. ${ }^{30}$ used Fourier amplitude distributions as snapshots for POD. In the current study, we are dealing with mainly broadband noise sources as well as intermittent events and, therefore, POD was applied in the temporal domain. The frequency information of dominant unsteady jet and shock dynamics is obtained by applying a Fourier transform to the POD time-coefficients.

Furthermore, the appropriate norm for capturing particular flow physics is not necessarily clear. Freund and Colonius ${ }^{29}$ have found that the effectiveness of a given representation of the initial flow data depends strongly upon the norm used and the data represented. For problems where the spatial dimension, $m$, is much greater than the number of available time-steps, $N$, it is more efficient to employ the "snapshot" method $^{31}$ for POD. Hence, in the current analysis the snapshot method is used. For more details about the POD snapshot method used here, see Chatterlee ${ }^{32}$ for a basic introduction to POD, and Rowley ${ }^{33}$ and Freund and Colonius ${ }^{29}$ on details on how it can be applied to fluid dynamics problems. In the current discussion, it is assumed that a vector of flow quantities, here $\vec{q}(\vec{x}, t)=\left(p^{\prime}, u^{\prime}, v^{\prime}, w^{\prime}, s^{\prime}\right)$, is defined over a region of interest $\Omega$ and additional weighting factors $\vec{\omega}$ weigh the contribution of the components of $\vec{q}(\vec{x}, t)$ 
in the vector norm,

$$
|\vec{q}|_{\Omega}^{2}=\int_{\Omega}\left(\sum_{k=1}^{N_{q}} \omega_{k} q_{k}^{2}\right) d \vec{x},
$$

where $N_{q}$ is the number of components of $\vec{q}$. By employing POD the series expansion converges optimally with respect to the $\mathrm{L}_{2}$-norm defined in Equation (9). Kinetic disturbance energy, the entropy fluctuation term, pressure or other combination of $\vec{q}$ can be used in the $\mathrm{L}_{2}$-norm by choosing the weighting vector as $\vec{\omega}=(0,1,1,1,0),(0,0,0,0,1)$, and $(1,0,0,0,0)$, respectively. In Brehm et al., ${ }^{34}$ it was shown that computing the most energetic $p^{\prime}$-based POD modes is useful for obtaining a general sense of the unsteady shock motion for jet impingement problems. In order to study the noise generation process $s^{\prime}$-based and $K^{\prime}$-based POD modes provide some idea of how noise is generated in the source region when considering that the general structure of Lighthill's stress tensor is composed of $\rho u_{i}^{\prime} u_{j}^{\prime}$ and the entropy fluctuation term $s^{\prime}$. More details about the computation of the POD modes and singular values associated with each mode are provided in appendix A.

\section{VI.A.1. Preliminary POD Results}

Figures 5 display the most energetic pressure-based POD mode shapes (a,c,e,f) and corresponding spectra of the time-coefficients $(b, d, f, h)$. Note that the POD analysis was conducted in a coordinate frame rotating with the front rotor. The tip vortex can be viewed as a steady mode in the rotating frame and is most dominant in the first two POD modes. The peak at shaft order 20 is associated with pressure wave generated by the aft rotor interacting with the front rotor and its tip vortices. In a fixed coordinate frame the aft blade generates pressure waves at shaft orders $n \times 10$, where $n$ is a positive integer value. In the contra-rotating frame (with the same rotational speed), the shaft orders are shifted to higher frequencies by a factor of 2 . Considering the mathematical foundation of the POD modes it is clear that a single POD mode is essentially spatially stationary. This means that at least two POD modes are required in order to mathematically describe a traveling wave in terms of POD modes. This is the reason why POD modes frequently occur in pairs. The third and fourth most dominant POD modes display the aforementioned property. The frequency spectra of the time-coefficients are almost identical and the POD mode pair physically describes the propagation of high-amplitude pressure waves.

\section{VI.B. Spectral Near-Field Analysis}

Figure 7 shows the most dominant blade-passing-frequencies (BPF) in the center-plane through the open rotor. Note that the results are expected to be significantly affected by the grid resolution. Hence, only preliminary conclusion can be drawn from the coarse grid simulation results. As one may expect, the most dominant pressure waves right in front of the open rotor are associated with the front rotor BPF1 while the aft rotor BPF2 remains dominant over a large region emerging from the back of the open rotor. Interestingly, a higher-order interaction $(\mathrm{BPF} 1+2 \times \mathrm{BPF} 2)$ is dominant in the wake. The unsteady tail shock located slightly downstream of the abrupt change of the hub geometry generates significant pressure waves and appears to be dominated by BPF1.

The noise generation process is further analyzed by considering the amplitude and phase plots at distinct frequencies. The amplitude distribution for BPF1 and BPF2 display large amplitudes at the front and aft blade set, respectively. The tip vortices from the front and aft blades remain dominant in the wake and an interaction with the tail shock can be noted. Another weaker shock is formed right behind the aft blade and it appears to be dominated by BPF2. The amplitude and phase plots for BPF $1+2 \times$ BPF 2 seem to highlight dominant pressure waves originating from a region right behind the aft rotor in front of the aforementioned weaker shock. 


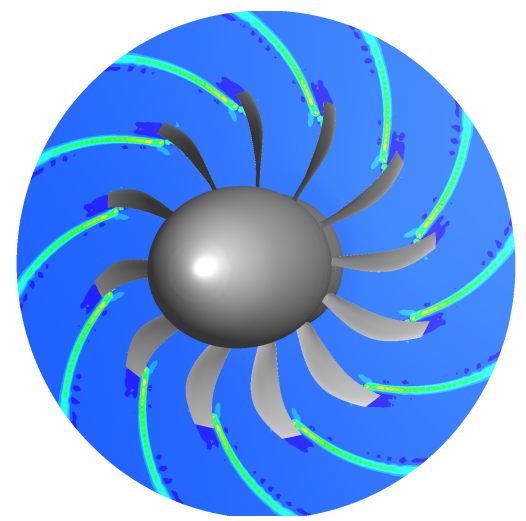

(a)

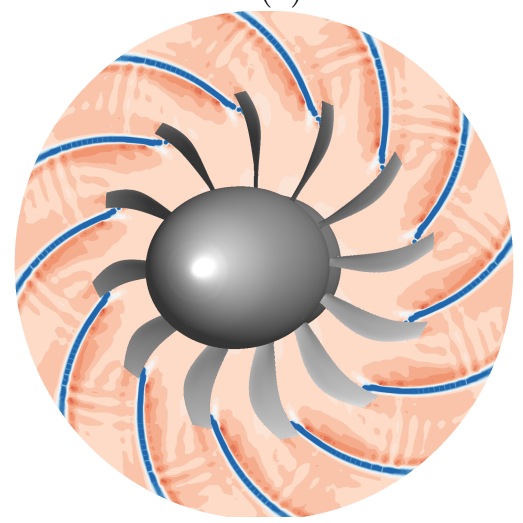

(c)

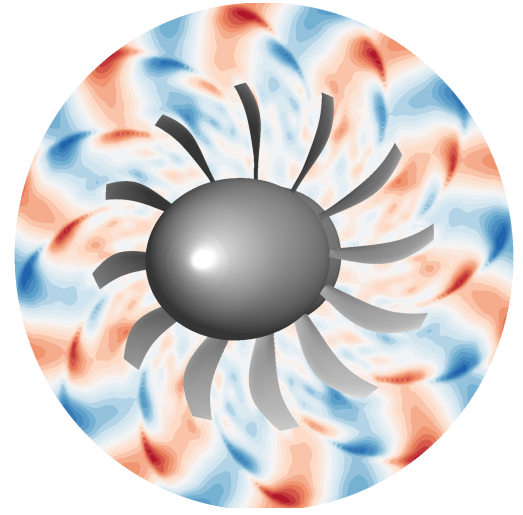

(e)

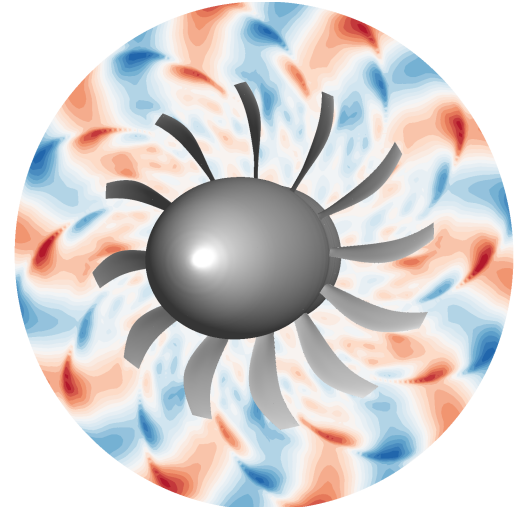

(g)

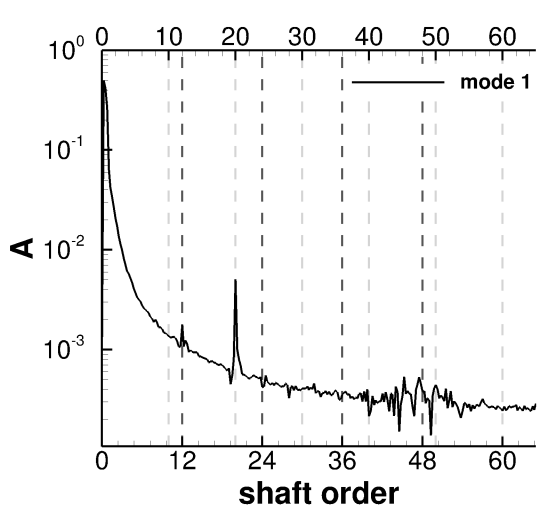

(b)

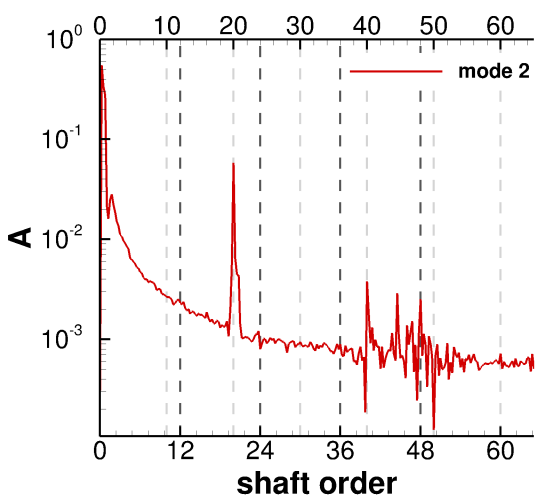

(d)

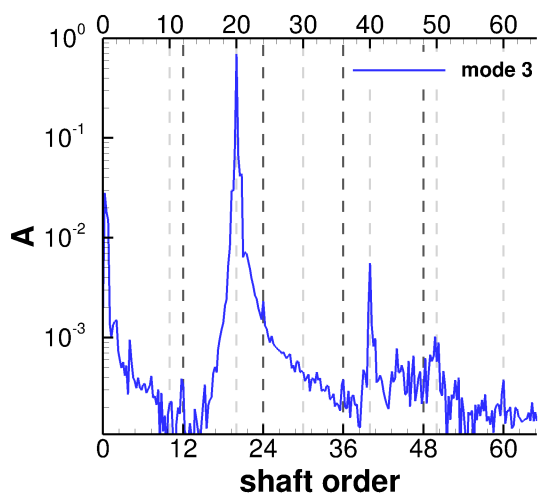

(f)

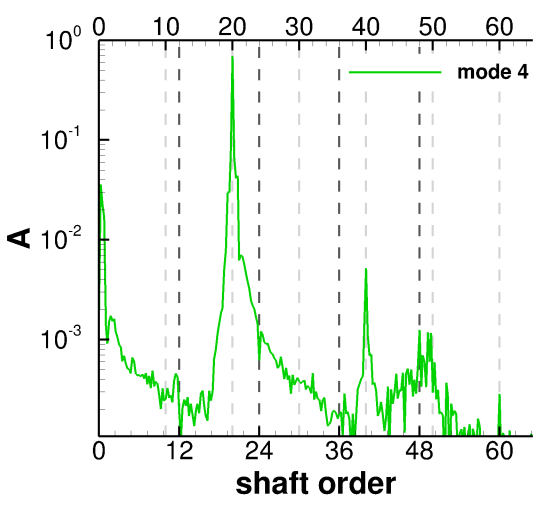

(h)

Figure 5. Most energetic pressure-based POD mode shapes (a,c,e,g) and corresponding spectra of the time-coefficients $(b, d, f, h)$ in a rotating frame with the front rotor. 

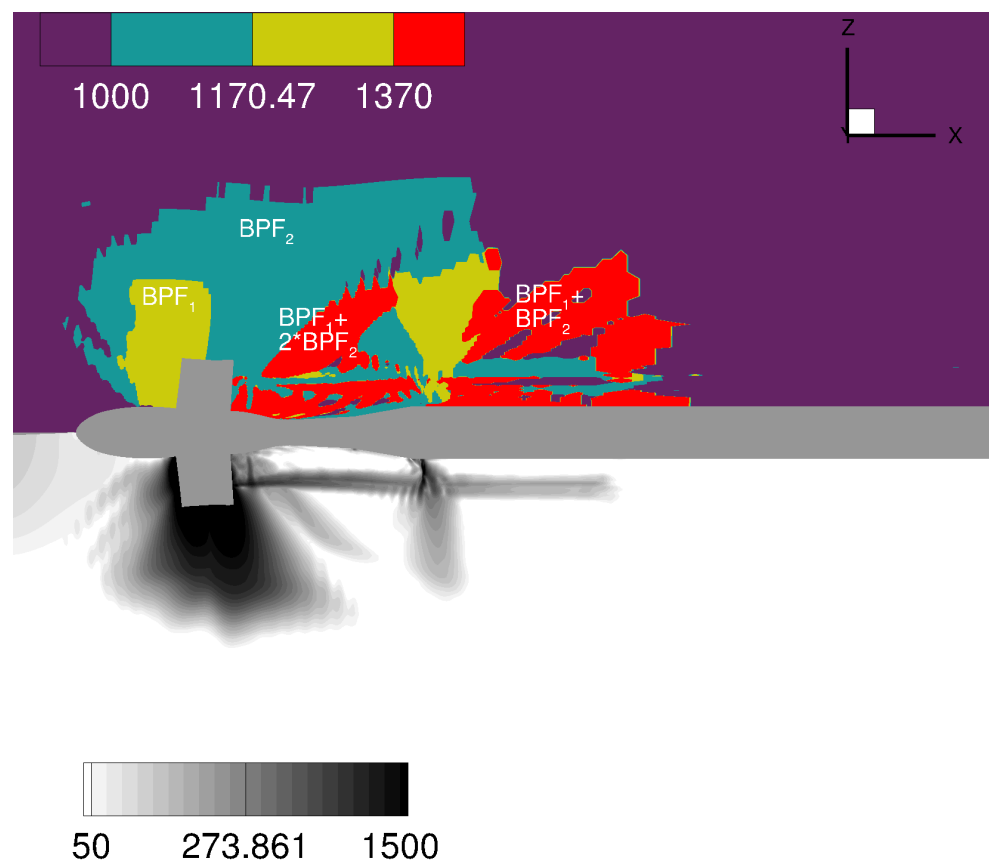

Figure 6. Most dominant frequencies (in $\mathrm{Hz}$ ) and corresponding Fourier amplitude displayed in the center-plane cutting through the open rotor. Note that the blade-passing-frequency of the front rotor is $\mathrm{BPF} 1=1370 \mathrm{~Hz}$ and $\mathrm{BPF} 2=1140 \mathrm{~Hz}$.

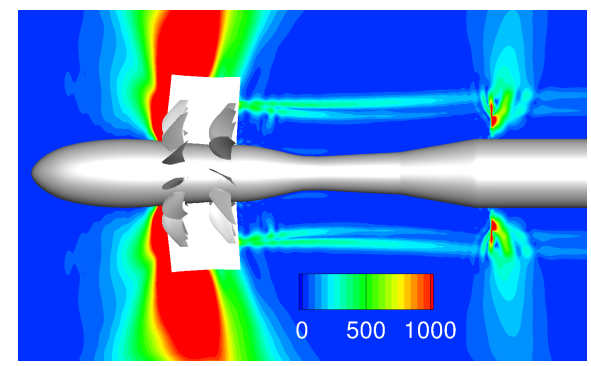

(a)

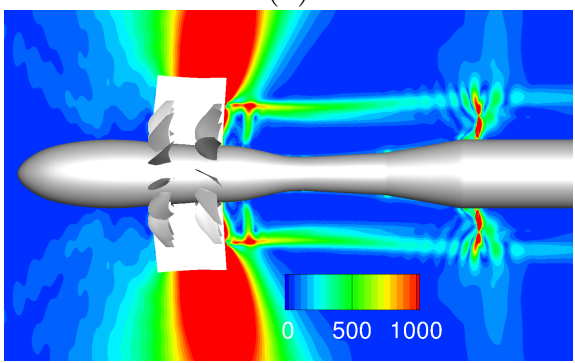

(c)

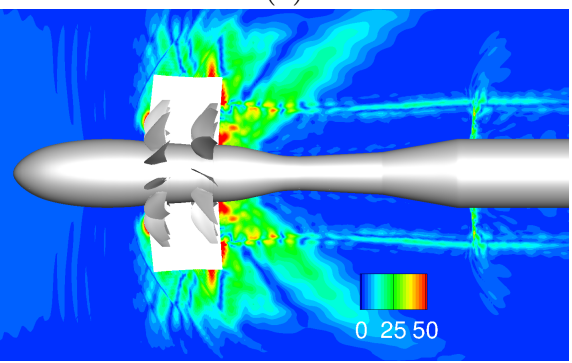

(e)

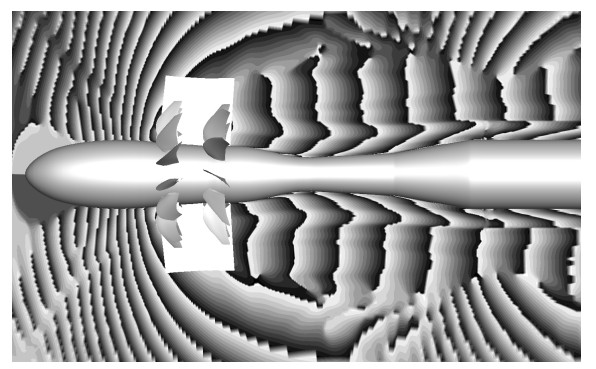

(b)

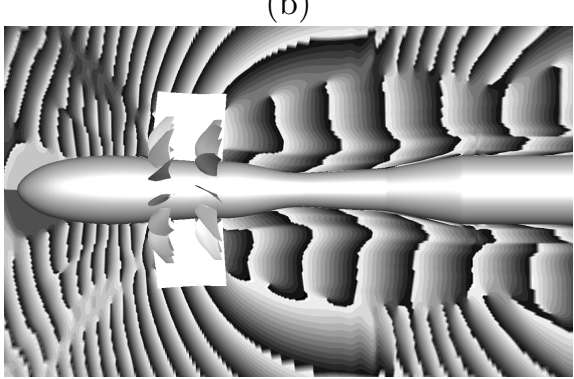

(d)

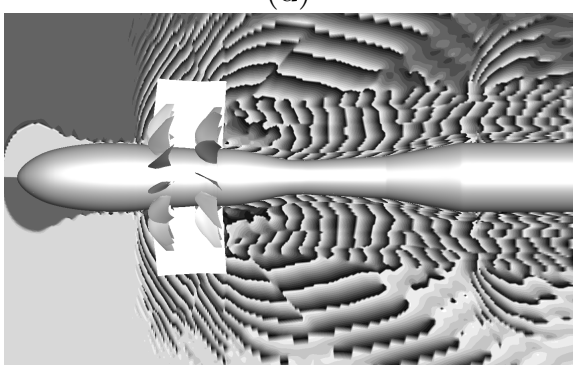

(f)

Figure 7. Amplitude and phase distributions at different blade-passing-frequencies: (a) BPF1, (b) BPF2, and (c) BPF1+2 $\times$ BPF2. 


\section{VI.C. Causality Method}

One of the key objectives of this work is to identify the dominant noise sources and uncover the relevant physical mechanisms responsible for noise generation. To investigate noise generation mechanisms, the dynamics of the unsteady flow field discussed in section IV need to be connected to the radiated sound field. Simultaneous visualizations of the instantaneous flow and sound fields can provide an idea on specific events that generate noise, see for example. ${ }^{35-37}$ In the current study, we employ a more direct approach by using the causality method, which relies on computing cross-correlations between flow quantities inside the open rotor flow field and the radiated acoustic pressure outside the non-linear flow regime. An attractive feature of this method is that the effects of scattering, absorption and refraction on sound radiation are automatically included by simultaneously extracting information from both the flow and acoustic fields. The present work is based upon the causality idea proposed in earlier works by. ${ }^{38-40}$ Computing the correlation between turbulent fluctuations inside the unsteady open rotor flow field and the radiated noise in the far field is the most direct and unambiguous way of identifying the relevant noise sources. The current theoretical framework is based on the acoustic analogy employed in Lighthill's equation (10). It is well-known that the decomposition proposed in Lighthill's analogy is non-unique. Flow-acoustic interactions are generally not distinguished from true acoustic sources. ${ }^{41}$ However, more elaborate formulations aimed at extending the acoustic analogy to account for the flow-acoustic interactions sacrifice the inherent simplicity of Lighthill's original approach. In the current paper, flow-acoustic interaction is not explicitly accounted for and the noise sources shall be interpreted in terms of Lighthill's sources. These source term, however, contains fluid dynamics that do not follow the homogeneous-medium second-order wave equation. Because flow-acoustic interactions have been demonstrated to be important in shear layers, the results need to be interpreted carefully. ${ }^{42,43}$

Lighthill's equation is defined as

$$
\frac{\partial \rho}{\partial t}-a_{\infty}^{2} \nabla^{2} \rho=\frac{\partial^{2} T_{i j}}{\partial x_{i} \partial x_{j}}
$$

where the right-hand-side term contains Lighthill's stress tensor $T_{i j}=\rho u_{i} u_{j}+\delta_{i j}\left(p-a_{\infty}^{2} \rho\right)$ absent the effect of viscosity, $u_{i}$ is the unsteady velocity field, and $p$ is the unsteady pressure field. In the current discussion, it is assumed that the viscous effects are negligible for the noise generation as well as feedback from the acoustic field to the source. It is important to remember that Lighthill's equation is simply a reformulation of the mass and momentum equations and is valid for every solution that obeys these equations. Lighthill's equation cannot distinguish between radiating and non-radiating disturbances. Theoretically, the relationship $a_{\infty}<\omega / \alpha$ can be used to separate the radiating and non-radiating parts, where $\omega$ is the angular frequency and $\alpha$ is the spatial wave number. In the current approach, we do not utilize this relationship explicitly and pursue a slightly different route to identify the radiating part of the solution. We follow the idea that multiplying with the acoustic pressure fluctuations in the far field acts simply as a filter operation, because sampling points located in the far field only sense the radiated part of disturbances. ${ }^{44}$

The most relevant parts of the causality approach are presented below. The theoretical foundation of our noise source identification strategy is laid out in more detail in Brehm et al. ${ }^{21}$ The right-hand side of equation (10) can be regarded as a forcing term to the wave equation. Acoustically, the source terms represent a distribution of quadrupoles embedded in an ambient medium whereby flow discontinuities are not explicitly contemplated. The desired solution at a far-field point is derived in terms of an integral equation after manipulating equation (10) and utilizing the time-domain free-space Green's function.

$$
\begin{aligned}
p^{\prime}\left(\boldsymbol{x}_{f}, t\right) & \approx \frac{x_{i} x_{j}}{4 \pi a_{\infty}^{2} r^{2}} \frac{\partial^{2}}{\partial t^{2}} \int_{V} \frac{T_{i j}\left(\boldsymbol{x}_{s}, t^{\prime}\right)}{\left|\boldsymbol{x}_{f}-\boldsymbol{x}_{s}\right|} d V+\frac{1}{4 \pi} \frac{\partial}{\partial t} \int_{S} \frac{\rho u_{i}}{\left|\boldsymbol{x}_{f}-\boldsymbol{x}_{s}\right|} n_{i} d S \\
& +\frac{x_{j}}{4 \pi r} \frac{\partial}{\partial t} \int_{S} \frac{\left(\delta_{i j} p+\rho u_{i} u_{j}\right)}{\left|\boldsymbol{x}_{f}-\boldsymbol{x}_{s}\right|} n_{i} d S .
\end{aligned}
$$

Following the analysis in Proudman ${ }^{45}$ as more recently revisited by Panda et al. ${ }^{44}$ we introduce the scalar component of the stress tensor $T_{r}$ that represent longitudinal quadrupoles. The scalar quantity is the fluctuating stress tensor component $T_{r}$ in the direction of the source point to the far-field observer location, i.e., $\boldsymbol{r}=\boldsymbol{x}_{f}-\boldsymbol{x}_{s}$. The acoustic intensity at a far-field point is finally obtained by multiplying equation (11) 
with the far-field acoustic pressure and computing the acoustic intensity,

$$
\begin{aligned}
\left\langle p^{\prime}, p^{\prime}\right\rangle\left(\boldsymbol{x}_{f}, \tau\right) & =\left\langle\frac{1}{4 \pi a_{0}^{2} \boldsymbol{r}} \int_{V} \frac{\partial^{2}}{\partial t^{\prime 2}} T_{r}\left(\boldsymbol{x}_{s}, t^{\prime}+\tau\right) d V, p^{\prime}\left(\boldsymbol{x}_{f}, t\right)\right\rangle \\
& =\frac{1}{4 \pi a_{0}^{2} \boldsymbol{r}} \int_{V} \frac{\partial^{2}}{\partial \tau^{2}} C_{T_{r}, p^{\prime}}\left(\boldsymbol{x}_{s}, \boldsymbol{x}_{f}, \tau\right) d V
\end{aligned}
$$

where the correlation function $C_{T_{r}, p^{\prime}}$ is evaluated after shifting the near field data $T_{r}$ by the propagation time or time delay $\tau$. Equation (12) provides an elegant way of linking the near field dynamics to the far-field acoustic pressure fluctuations. Applying such a cross-correlation is extremely powerful since it naturally separates the hydrodynamic and acoustic fluctuations. ${ }^{44}$ To avoid the computation of the second derivatives in time, we apply a temporal Fourier transform to equation (12) and find

$$
P S D_{p^{\prime}}\left(\boldsymbol{x}_{f}, f\right)=-\frac{\pi f^{2}}{r a_{\infty}^{2}} \int_{V} C S D_{T_{r}, p^{\prime}}\left(\boldsymbol{x}_{f}, \boldsymbol{x}_{s}, f\right) d V
$$

where the auto-correlation function of $p^{\prime}$ is transformed to the power spectral density $P S D_{p^{\prime}}$ and the crosscorrelation function between $T_{r}$ and $p^{\prime}$ turns into the cross-spectral density $C S D_{T_{r}, p^{\prime}}$.

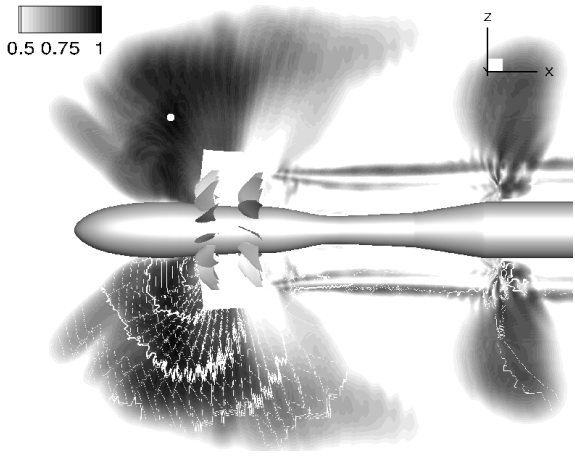

(a)

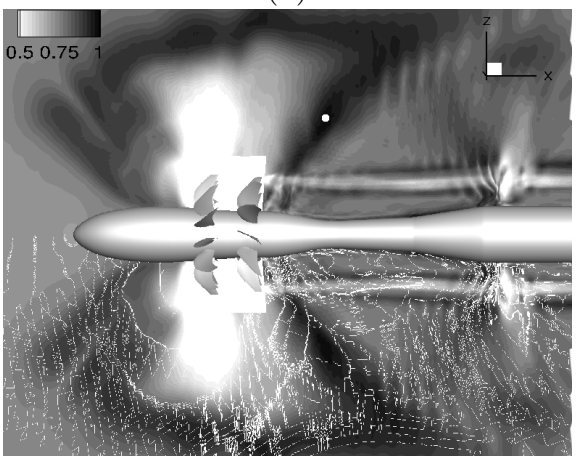

(c)

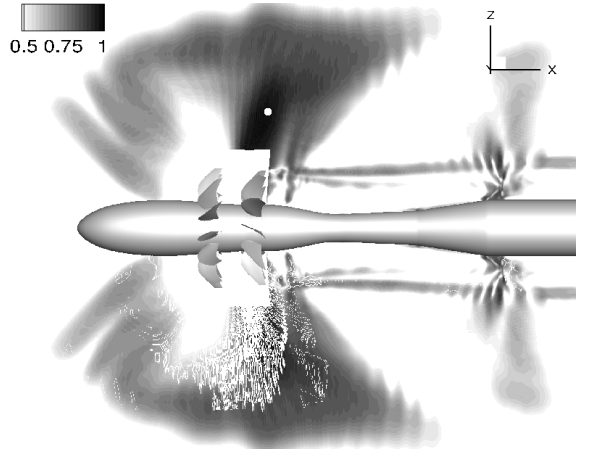

(b)

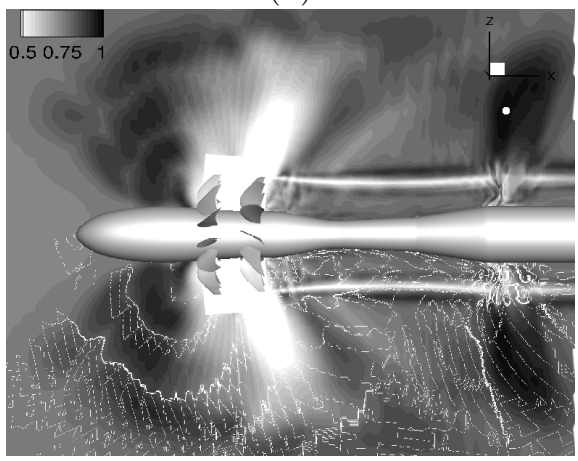

(d)

Figure 8. Normalized cross-correlation of acoustic pressure at different sample points marked with white filled circle $(\bullet)$ and pressure on the sampling plane. Lines of constant time-delay are shown in the lower half of these figures with white solid lines.

This method has previously been applied to a jet impingement problem discussed in Brehm et al. ${ }^{21}$ and it was shown to be very useful in studying the noise generation process. Figure 8 displays preliminary results utilizing normalized cross-correlations of acoustic pressure at different sample points marked with white filled circles and pressure on the sampling plane. The cross-correlation function varies from 0 to 1. Note the analysis employing the causality method will be significantly extended utilizing different terms of Lighthill's stress tensor, normalized and non-normalized correlations. An important aspect of understanding the noise generation process is to determine which of the source terms are most dominant. Furthermore, to analyze turbo-machinery noise we will also study the contributions from the surface integrals in equation (11) and compare this result to traditional lower-order noise prediction methods that are based on a similar acoustic analogy. This way we will be able to point to weaknesses of these methods and possibly suggest improvements. 


\section{Summary and Outlook}

In the present paper, we employ a higher-order accurate immersed boundary method to simulate the highly complex flow field around an open rotor. The current abstract provides an overview of the different topics that will be covered in more detail in the final paper. Two key aspects will be addressed in this paper: (1) the challenges of simulating the open rotor with our higher-order immersed boundary method and (2) a detailed analysis of the most dominant noise generation mechanisms of the open rotor propulsion system. The first part of the paper deals with the numerical simulation approach and the peculiarities of the immersed boundary method. In order to gain confidence in our simulation approach we are providing a detailed comparison with experimental data. In the second part of the paper, several post-processing tools, such as Fourier transforms, POD, and the causality method, are used to obtain a detailed understanding of the noise generation process. Finally, we want to emphasize that the results presented in the current abstract are based on a coarse grid simulation. The final paper will include a grid resolution study considering additional medium and fine grid solutions.

\section{Appendix A: Computation of POD Modes}

The POD eigenmodes are computed as

$$
\vec{\psi}^{(n)}(\vec{x})=\sum_{i=0}^{N} r_{i}^{(n)} \vec{q}\left(\vec{x}, t_{i}\right)
$$

where the subscript $i$ indicates the time-step $(i=0$ to $N), \vec{q}\left(\vec{x}, t_{i}\right)$ is the vector of flow quantities at timestep $i$, and $r_{i}^{(n)}$ are individual elements of $\vec{r}^{(n)}$. The right eigenvectors, $\vec{r}^{(n)}$, are solutions to the algebraic eigenvalue problem:

$$
\mathbf{C} \vec{r}^{(n)}=\lambda^{(n)} \vec{r}^{(n)},
$$

where the correlation tensor $\mathbf{C}$ is defined in index notation as

$$
C_{i j}=\frac{1}{N+1}\left[\vec{q}\left(\vec{x}, t_{i}\right), \vec{q}\left(\vec{x}, t_{j}\right)\right] .
$$

Here, the brackets denote the inner product $[\vec{q}, \vec{q}]=\int_{\Omega}\left(\sum_{k=0}^{N_{q}} \omega_{k} q_{k}^{2}\right) d \vec{x}$ (see Equation (9)). The eigenfunctions are normalized with their corresponding eigenvalues $\left[\vec{\psi}^{(n)}, \vec{\psi}^{\left(n^{\prime}\right)}\right]=\delta_{n n^{\prime}} \lambda^{(n)}$, and the time coefficients are computed as

$$
a^{(n)}(t)=\frac{1}{\lambda^{(n)}}\left[\vec{q}(\vec{x}, t), \vec{\psi}^{(n)}(\vec{x})\right] .
$$

The flow field can be reconstructed/recovered from the eigenmodes and time coefficients:

$$
\vec{q}(\vec{x}, t) \approx \sum_{n=0}^{I} a^{(n)}(t) \vec{\psi}^{(n)}(\vec{x}) .
$$

The POD modes are orthogonal to each other and sorted by their respective energy norm (see Equation (9)) contents, whereby the drop-off in mode energy toward the higher mode numbers is typically significant. Therefore, a small number of modes, I, will suffice for capturing a large percentage of the flow's energy defined in Equation (9). This is particularly the case when the flow dynamics are governed by large energetic structures of organized (or coherent) fluid motion (such as in pure two-dimensional simulations).

\section{References}

\footnotetext{
${ }^{1}$ Spalart, P., Travin, A., Shur, M., and Strelets, M., "Initial Noise Predictions for Open Rotors Using First Principles," 16th AIAA/CEAS Aeroacoustics Conference, Stockholm, Sweden, June 2010, AIAA-2010-3793.

${ }^{2}$ Rebecca Busch, E., Kebler, M., and Kramer, E., "Aeroacoustics of a High-Fidelity CFD Calculation of a CounterRotating Open Rotor in Take-Off Conditions," 19th AIAA/CEAS Aeroacoustics Conference, Berlin, Germany, May 2013, AIAA-2013-2202.
} 
${ }^{3}$ Ben Nasr, N., Ortun, B., Chelius, A., and Canard-Caruana, S., "Assessment of advanced grid strategies for CFD on open rotor applications," 49th AIAA/ASME/SAE/ASEE Joint Propulsion Conference, San Jose, CA, July 2013, AIAA-2013-3800.

${ }^{4}$ Node-Langlois, T., Wlassow, F., Languille, V., Colin, Y., Caruelle, B., Gill, J., Chen, X., Zhang, X., and Parry, A., "Prediction of Contra-Rotating Open Rotor broadband noise in isolated and installed configurations," 20th AIAA/CEAS Aeroacoustics Conference, Atlanta, Georgia, June 2014, AIAA-2014-2610.

${ }^{5}$ Envia, E., "Contra-Rotating Open Rotor Tone Noise Prediction," 20th AIAA/CEAS Aeroacoustics Conference, Atlanta, Georgia, June 2014, AIAA-2014-2606.

${ }^{6}$ Peskin, C., "Numerical Analysis of Blood Flow in the Heart," J. Comput. Phys., Vol. 25, 1977, pp. 220-252.

${ }^{7}$ Peskin, C. S., "The Immersed Boundary Method," Acta Numerica, Cambridge University Press, 2002, pp. 1-39.

${ }^{8}$ Goldstein, D., Handler, R., and Sirovich, L., "Modeling a Non-Slip Flow Boundary with an External Force Field," J. Comp. Phys., Vol. 105, 1993, pp. 354-366.

${ }^{9}$ LeVeque, R. J., Time-Split Methods for Partial Differential Equations, Ph.D. thesis, Stanford University, 1982.

${ }^{10}$ Wiegmann, A. and Bube, K., "The Explicit-Jump Immersed Interface Method: Finite Difference Methods for PDEs with Piecewise Smooth Solutions," SIAM J. Numer. Anal., Vol. 31(4), 1992, pp. 1019-1044.

${ }^{11}$ Linnick, M. and Fasel, H., "A High-Order Immersed Interface Method for Simulating Unsteady Incompressible Flows on Irregular Domains," J. Comput. Phys, Vol. 204, 2004, pp. 157-192.

12 Johansen, H. and Collela, P., "A Cartesian Grid Embedded Boundary Method for Poisson's Equation on Irregular Domains," Journal of Computational Physics, Vol. 147, 1998, pp. 60-85.

${ }^{13}$ Mittal, R. and Iaccarino, G., "Immersed Boundary Methods," Annu. Rev. Fluid Mech., Vol. 37, 2005, pp. $239-261$.

${ }^{14}$ Zhong, X., "A New High-Order Immersed Interface Method for Solving Elliptic Equations with Embedded Interface of Discontinuity," Journal of Computational Physics, Vol. 225, 2007, pp. 1066-1099.

${ }^{15}$ Duan, L., Wang, X., and Zhong, X., "A High-Order Cut-Cell Method for Numerical Simulation of Hypersonic BoundaryLayer Instability with Surface Roughness," Journal of Computational Physics, Vol. 229, 2010, pp. 7207-7237.

${ }^{16}$ Kiris, C., Barad, M., Housman, J., Sozer, E., Brehm, C., and Moini-Yekta, S., "The LAVA Computational Fluid Dynamics Solver," 52st AIAA Aerospace Sciences Meeting, National Harbor, Maryland, January 13-17, 2014, AIAA-2014-0070.

${ }^{17}$ Nemec, M. and Aftosmis, M., "Aerodynamic Shape Optimization Using a Cartesian Adjoint Method and CAD Geometry," 24th AIAA Applied Aerodynamics Conference, AIAA 2006-3456, 2006.

${ }^{18}$ Mittal, R., Dong, H., Bozkurttas, M., Najjar, F., Vargas, A., and von Loebbecke, A., "A versatile sharp inerface immersed boundary method for incompressible flows with complex boundaries," Journal of Computational Physics, Vol. 227, 2008, pp. $4825-4852$.

${ }^{19}$ Tezduyar, T., "Finite Element Methods for Flow Problems with Moving Boundaries and Interfaces," Arch. Comput. Methods Eng., Vol. 8, 2001, pp. 83-130.

${ }^{20}$ Brehm, C. and Fasel, H., "A novel concept for the design of immersed interface methods," Journal of Computational Physics, Vol. 242, No. 0, 2013, pp. $234-267$.

21 "A locally stabilized immersed boundary method for the compressible NavierStokes equations," Journal of Computational Physics, Vol. 295, No. 0, 2015, pp. $475-504$.

${ }^{22}$ Laible, A. C. and Fasel, H. F., "Temporal Direct Numerical Simulations of Oblique Breakdown for a Cone at Mach 3.5," 49th AIAA Aerospace Sciences Meeting, 2011-0209, 2011.

${ }^{23}$ Zhong, X., "High-Order Finite-Difference Schemes for Numerical Simulation of Hypersonic Boundary-Layer Transition," J. Comp. Phys., Vol. 144, 1998, pp. 662-709.

${ }^{24}$ Visbal, M. and Gaitonde, D., "High-order accurate methods for unsteady vortical flows on curvilinear meshes," AIAA 1998-0131, 1998.

${ }^{25} \mathrm{Shu}, \mathrm{C}$. and Osher, S., "Efficient implementation of essentially non-oscillatory shock-capturing schemes," Journal of Computational Physics, Vol. 77, No. 2, 1988, pp. 439-471.

${ }^{26}$ Brehm, C., Barad, M., Housman, J., and Kiris, C., "A Comparison of Higher-Order Finite Difference Shock Capturing Schemes," Computer and Fluids, submitted, 2014.

${ }^{27}$ van Leer, B., "Flux Splitting for the Euler Equations," International Conference on Numerical Methods in Fluid Dynamics, Vol. 170, 1982, pp. 507-512.

${ }^{28}$ Lumley, J., "The structure of inhomogeneous turbulent flows," Atm. Turb. And Radio Wave Prop., Nauka, Moscow and Toulouse, France, eds. Yaglom and Tatarsky, 1967, pp. 166-178.

${ }^{29}$ Freund, J. B. and Colonius, T., "POD Analysis of Sound Generation by a Turbulent Jet," AIAA 2002-0072, 40th AIAA Aerospace Sciences Meeting and Exhibit, 2002, pp. 1-9.

${ }^{30}$ Suzuki, T., Bodony, D., Ryu, J., and Lele, S. K., "Noise Sources of High-Mach-number Jets at Low Frequencies Studied with a Phased-array Approach Based on LES Database," Annual research briefs, Center for Turbulence Research, NASA Ames and Stanford University, 2007, http://www.stanford.edu/group/ctr/ResBriefs/ARB07.html.

${ }^{31}$ Sirovich, L., "Turbulence and the Dynamics of Coherent Structures," Quarterly of Applied Mathematics, Vol. XLV(3), 1987, pp. 561-590.

${ }^{32}$ Chatterjee, A., "An introduction to the proper orthogonal decomposition," Current science, Vol. 78, No. 7, 2000, pp. 808817.

${ }^{33}$ Rowley, C. W., Modeling, simulation, and control of cavity flow oscillations, Ph.D. thesis, California Institute of Technology, July 2001.

${ }^{34}$ Brehm, C., Sozer, E., Moini-Yekta, S., Housman, J., Barad, M., Kiris, C., Vu, B., and Parlier, C., "Computational Prediction of Pressure Environment in the Flame Trench," AIAA Paper, June 24-27, San Diego, CA, 2013.

${ }^{35}$ Hileman, J. I., Thurow, B. S., Caraballo, E. J., and Samimy, M., "Large-scale structure evolution and sound emission in high-speed jets: real-time visualization with simultaneous acoustic measurements," Journal of Fluid Mechanics, Vol. 544, 2005, pp. 277-307. 
${ }^{36}$ Bogey, C. and Bailly, C., "LES of High Reynolds, High Subsonic Jet: Effects of the Inflow Conditions on Flow and Noise," AIAA Paper No. 2003-3170, May, 2003.

${ }^{37}$ Hileman, J. and Samimy, M., "Turbulence structures and the acoustic far field of a Mach 1.3 jet," AIAA journal, Vol. 39, No. 9, 2001, pp. 1716-1727.

${ }^{38}$ Siddon, T., "Noise source diagnostics using causality correlations," AGARD CP, Vol. 131, 1973, pp. 7-1.

${ }^{39}$ Seiner, J. M., "The distribution of jet source strength intensity by means of a direct correlation technique," 1974.

${ }^{40}$ Rackl, R. and Siddon, T., "Causality correlation analysis of flow noise with fluid dilatation as source fluctuation," The Journal of the Acoustical Society of America, Vol. 65, No. 5, 1979, pp. 1147-1155.

${ }^{41}$ Freund, J., "Noise sources in a low-Reynolds-number turbulent jet at Mach 0.9," Journal of Fluid Mechanics, Vol. 438, 2001, pp. 277-305.

${ }^{42}$ Goldstein, M., "The low frequency sound from multipole sources in axisymmetric shear flows, with applications to jet noise," Journal of Fluid Mechanics, Vol. 70, No. 03, 1975, pp. 595-604.

${ }^{43}$ Goldstein, M., "High frequency sound emission from moving point multipole sources embedded in arbitrary transversely sheared mean flows," Journal of Sound and Vibration, Vol. 80, No. 4, 1982, pp. 499-522.

${ }^{44}$ Panda, J., Seasholtz, R., and Elam, K., "Investigation of noise sources in high-speed jets via correlation measurements," Journal of Fluid Mechanics, Vol. 537, 2005, pp. 349-385.

${ }^{45}$ Proudman, I., "The generation of noise by isotropic turbulence," Proceedings of the Royal Society of London. Series A. Mathematical and Physical Sciences, Vol. 214, No. 1116, 1952, pp. 119-132. 